\title{
Fatores associados ao desmame precoce no Brasil: uma revisão integrativa
}

\author{
Factors associated with early weaning in Brazil: an integrative review \\ Factores asociados con el destete temprano en Brasil: una revisión integradora
}

Recebido: 31/12/2020 | Revisado: 02/01/2021 | Aceito: 25/01/2021 | Publicado: 31/01/2021

\author{
Natália Borba Cavalcanti \\ ORCID: https://orcid.org/0000-0001-7052-8490 \\ Universidade Católica de Pernambuco, Brasil \\ E-mail: cavalcantii.natalia@hotmail.com \\ Ana Carla Macedo da Silva \\ ORCID: https://orcid.org/0000-0003-0521-8909 \\ Universidade Católica de Pernambuco, Brasil \\ E-mail: Carlamacedo05@gmail.com \\ José William Araújo do Nascimento \\ ORCID: https://orcid.org/0000-0002-1844-1117 \\ Universidade Federal de Pernambuco, Brasil \\ E-mail: jwanb4321@gmail.com
}

\begin{abstract}
Resumo
Objetivo: Identificar na literatura científica os fatores que contribuem para o desmame precoce no Brasil. Metodologia: Revisão integrativa realizada na Biblioteca Virtual em Saúde, que permite o acesso simultâneo às bases de dados eletrônicas BDENF, IBECS e LILACS, por meio dos seguintes descritores: aleitamento materno, desmame, lactente, lactante, cuidados do lactente e saúde da criança. Resultados: 12 artigos compuseram a amostra final deste estudo, de modo que os fatores associados ao desmame precoce mais frequentes foram uso da chupeta (n: 03), escolaridade materna (n: 02), crença materna na falta de leite (n: 02), trabalhar fora do lar (n: 02), uso da mamadeira (n: 02), tabagismo materno (n: 01), ser primípara (n: 01), classe econômica (n: 01) e estado civil (n: 01). Conclusão: Os fatores associados ao desmame precoce encontrados nos estudos realizados no Brasil estão relacionados a hábitos de vida e crença, bem como a características socioeconômicas das mães, o que pode gerar prejuízos a saúde tanto do lactente como da lactante.
\end{abstract}

Palavras-chave: Aleitamento materno; Desmame precoce; Promoção da saúde.

\begin{abstract}
Objective: To identify in the scientific literature the factors that contribute to early weaning in Brazil. Methodology: Integrative review carried out at the Virtual Health Library, which allows simultaneous access to the electronic databases BDENF, IBECS and LILACS, through the following descriptors: breastfeeding, weaning, infant, lactating, infant care and child health. Results: 12 articles made up the final sample of this study, so that the most frequent factors associated with early weaning were pacifier use (n: 03), maternal education (n: 02), maternal belief in lack of milk (n: 02 ), working outside the home (n: 02), bottle feeding (n: 02), maternal smoking (n: 01), being primiparous (n: 01), economic class (n: 01) and marital status (n: 01). Conclusion: The factors associated with early weaning found in studies carried out in Brazil are related to life habits and belief, as well as to the socioeconomic characteristics of the mothers, which can cause damage to the health of both the infant and the infant.
\end{abstract}

Keywords: Breastfeeding; Early weaning; Health promotion.

\section{Resumen}

Objetivo: Identificar en la literatura científica los factores que contribuyen al destete temprano en Brasil. Metodología: Revisión integradora realizada en la Biblioteca Virtual en Salud, que permite el acceso simultáneo a las bases de datos electrónicas BDENF, IBECS y LILACS, a través de los siguientes descriptores: lactancia materna, destete, lactante, lactancia, atención infantil y salud infantil. Resultados: 12 artículos conformaron la muestra final de este estudio, por lo que los factores más frecuentes asociados al destete temprano fueron el uso de chupete (n: 03), educación materna (n: 02), creencia materna en la falta de leche (n: 02 ), trabajo fuera del hogar (n: 02), alimentación con biberón (n: 02), tabaquismo materno (n: 01), ser primíparas (n: 01), clase económica (n: 01) y estado civil (n: 01). Conclusión: Los factores asociados con el destete temprano encontrados en estudios realizados en Brasil están relacionados con los hábitos de vida y creencias, así como con las características socioeconómicas de las madres, que pueden dañar la salud tanto del lactante como de la madre lactante.

Palabras clave: Lactancia materna; Destete temprano; Promoción de la salud. 


\section{Introdução}

O leite materno é o alimento ideal para o crescimento e desenvolvimento saudável do lactente, exercendo influência biológica e emocional sobre a saúde da mãe e do bebê. Oferece à criança todos os nutrientes que necessita, tais como: proteína, gorduras, lactose, vitaminas, ferro, água, sais minerais, cálcio, fosfato, lipases e fatores anti-infecciosos (Silva et al., 2017). A Organização Mundial de Saúde (OMS) preconiza que o aleitamento materno seja oferecido de forma exclusiva até o sexto mês de vida do bebê e complementado até os dois anos ou mais (WHO, 2020).

Além de atender às necessidades nutricionais do lactente, o aleitamento materno reduz a mortalidade, morbidade e hospitalização de crianças por doenças infecciosas, como diarreia, pneumonia e otite (Victora et al., 2016; Buccini et al., 2017).

No entanto, apesar dos benefícios bem reconhecidos do aleitamento materno exclusivo (AME), as taxas de amamentação exclusiva aqui no Brasil estão longe de atender as recomendações da OMS. Uma pesquisa nacional apontou que $41 \%$ das crianças menores de 6 meses foram amamentadas exclusivamente (Brasil, 2015). No mundo, dados da OMS quanto ao aleitamento materno exclusivo em menores de seis meses se assemelham ao do Brasil (WHO, 2018). Consequentemente o desmame precoce representam um grave problema de saúde pública e está associado a fatores sociais, culturais e econômicos (Lopes, 2016).

Dentre as complicações do desmame estão: síndrome de morte súbita infantil; desenvolvimento de eczema; problemas digestivos decorrentes do uso precoce de fórmulas ou de alimentos sólidos; maior risco de desenvolver à obesidade infantil e doenças crônico-degenerativas como hipertensão arterial, diabetes mellitus e doenças cardíacas (D'Auria et al., 2018; Feitosa et al., 2020).

Nesta perspectiva de prevenção aos agravos da saúde da criança, o enfermeiro tem fundamental importância na promoção do aleitamento materno desde o período do pré-natal, no puerpério na puericultura. Este profissional contribui, por meio da educação em saúde, oferecendo suporte emocional frente aos obstáculos que podem surgir, orientando as nutrizes acerca dos benefícios do aleitamento materno, das técnicas corretas de amamentação e ordenha manual. Além disso, é de extrema importância que os enfermeiros organizem grupos de apoio a amamentação, estimulando a participação das nutrizes para que o aleitamento materno não seja interrompido precocemente (Araújo et al., 2020).

Tendo em vista o impacto do desmame precoce na saúde da criança, desvelar os fatores que contribuem para este fenômeno irá proporcionar um direcionamento adequado no cuidado e acompanhamento por parte do enfermeiro no período pré-natal, pós-parto e na puericultura. Com isso, favorecendo o bem-estar do bebê e da mãe, considerando suas vivências e diminuindo assim as complicações ocasionadas pelo desmame precoce.

A proposta para realização desta pesquisa justifica-se pela importância de identificar os fatores associados ao desmame precoce, proporcionando um escopo de evidências sólidas para um direcionamento adequado no cuidado e acompanhamento pelo enfermeiro no período pré-natal, pós-parto e na puericultura. Desta forma esta pesquisa objetivou identificar na literatura científica os fatores que contribuem para o desmame precoce no Brasil.

\section{Metodologia}

Foi realizado um estudo de revisão integrativa da literatura de escopo quantitativo, que analisa uma problemática por meio da análise de resultados encontrados em artigos, permitindo a compreensão de um problema, bem como como a incorporação de evidências da prática clínica (Pereira, 2011; Pereira, 2018).

Esta pesquisa foi realizada a partir das seguintes etapas: 1) Definição da questão norteadora e objetivo da pesquisa; 2) Estabelecimento dos critérios de inclusão e exclusão/amostragem dos estudos; 3) Busca na literatura; 4) Categorização e análise dos estudos; 5) Apresentação e discussão dos resultados da amostra e 6) Apresentação e síntese do conhecimento 
(Souza et al., 2010).

A investigação dos estudos ocorreu nas seguintes bases de dados: Base de Dados de Enfermagem (BDENF), Literatura Latino-americana e do Caribe em Ciências da Saúde (LILACS) e Índice Bibliográfico Español en Ciencias de la Salud (IBECS), por intermédio da Biblioteca Virtual de Saúde (BVS), uma vez que esta permite uma busca simultânea nas principais fontes nacionais e internacionais. A busca ocorreu no período de agosto a novembro de 2020.

Para realizar as buscas nas bases de dados foi feita uma consulta ao Descritores em Ciências da Saúde (DeCS) e utilizados os seguintes descritores: aleitamento materno, desmame, lactente, lactante, cuidados do lactente e saúde da criança.

Os estudos foram selecionados mediante os seguintes critérios de inclusão: artigos disponíveis eletronicamente nas bases de dados selecionadas, publicados em português, inglês ou espanhol entre 2015 a 2020, pesquisas cujos locais de realização tenham sido no Brasil e estudos que apresentem dados estatísticos significativos que evidenciem o fator associado ao desmame precoce.

Foram excluídos os artigos duplicados nas bases de dados (duplicatas), os que não se propunham a discutir o desmame precoce, os estudos de revisão (narrativa, integrativa e sistemática), teses, dissertações e trabalho de conclusão de curso, capítulos de livro, artigos de reflexão, reportagens e editorias de jornais sem caráter científico.

A coleta de dados foi realizada na BVS utilizando o formulário avançado com as combinações dos descritores. A busca deu-se início através da aplicação dos descritores de forma individualizada e em seguida foram realizados os cruzamentos utilizando os operadores booleanos "AND" e "OR". Após os cruzamentos dos dados, que podem ser consultados no Quadro 1, foram encontradas 184 publicações, sendo 119 na LILACS, 58 na BDENF e 7 na IBECS.

Quadro 1. Estratégias de busca utilizadas nas bases de dados, Recife (PE), Brasil, 2020.

\begin{tabular}{|l|l|l|l|l|}
\hline DESCRITORES & LILACS & BDENF & IBECS & TOTAL \\
\hline $\begin{array}{l}\text { ("Aleitamento materno") AND } \\
\text { ("lactente" OR lactante") AND } \\
\text { ("desmame") }\end{array}$ & 53 & 24 & 6 & 83 \\
\hline $\begin{array}{l}\text { ("Aleitamento materno") AND } \\
\text { ("lactente" OR "cuidado do lactente" } \\
\text { OR "saúde da criança") AND } \\
\text { ("desmame") }\end{array}$ & 66 & 34 & 1 & 101 \\
\hline TOTAL & 119 & 58 & 7 & 184 \\
\hline
\end{tabular}

Fonte: Autores.

Para garantir o registro do conjunto de informações relevantes ao tema, foi utilizado instrumento proposto por Souza et al (2018) adaptado para este estudo com as seguintes variáveis: dados de identificação geral (título, autores, periódico, ano de publicação, país de origem do estudo, qualis do periódico e base de dados), delineamento metodológico (tipo/abordagem do estudo e nível de evidência) e principais resultados específicos.

Os artigos selecionados foram submetidos à classificação do nível de evidência, a partir do instrumento de Classificação Hierárquica das Evidências para Avaliação dos Estudos. Segundo esta classificação, os níveis I e II são considerados evidências fortes, III e IV moderadas e V a VII fracas (Stillwell et al., 2010).

Após a aplicação dos filtros de pesquisa, inicialmente foram encontrados 184 artigos, sendo 119 indexados na LILACS, 58 na BDENF e 07 na IBECS, de modo que os artigos duplicados foram registrados apenas uma vez, totalizando em 138 publicações. 
Conforme é explicitado na Figura 1, após a leitura dos títulos e resumos simultaneamente foram excluídas 79 publicações por não atenderem aos critérios de elegibilidade. Sendo assim, 59 artigos foram selecionados para leitura na íntegra, porém 47 foram excluídos por não atenderem aos critérios adotados. Assim, a amostra final foi constituída por doze artigos. Do total de doze artigos analisados, onze estavam indexados na LILACS e um na BDENF.

Figura 1. Fluxograma do processo de seleção do estudo, Recife (PE), Brasil, 2020.

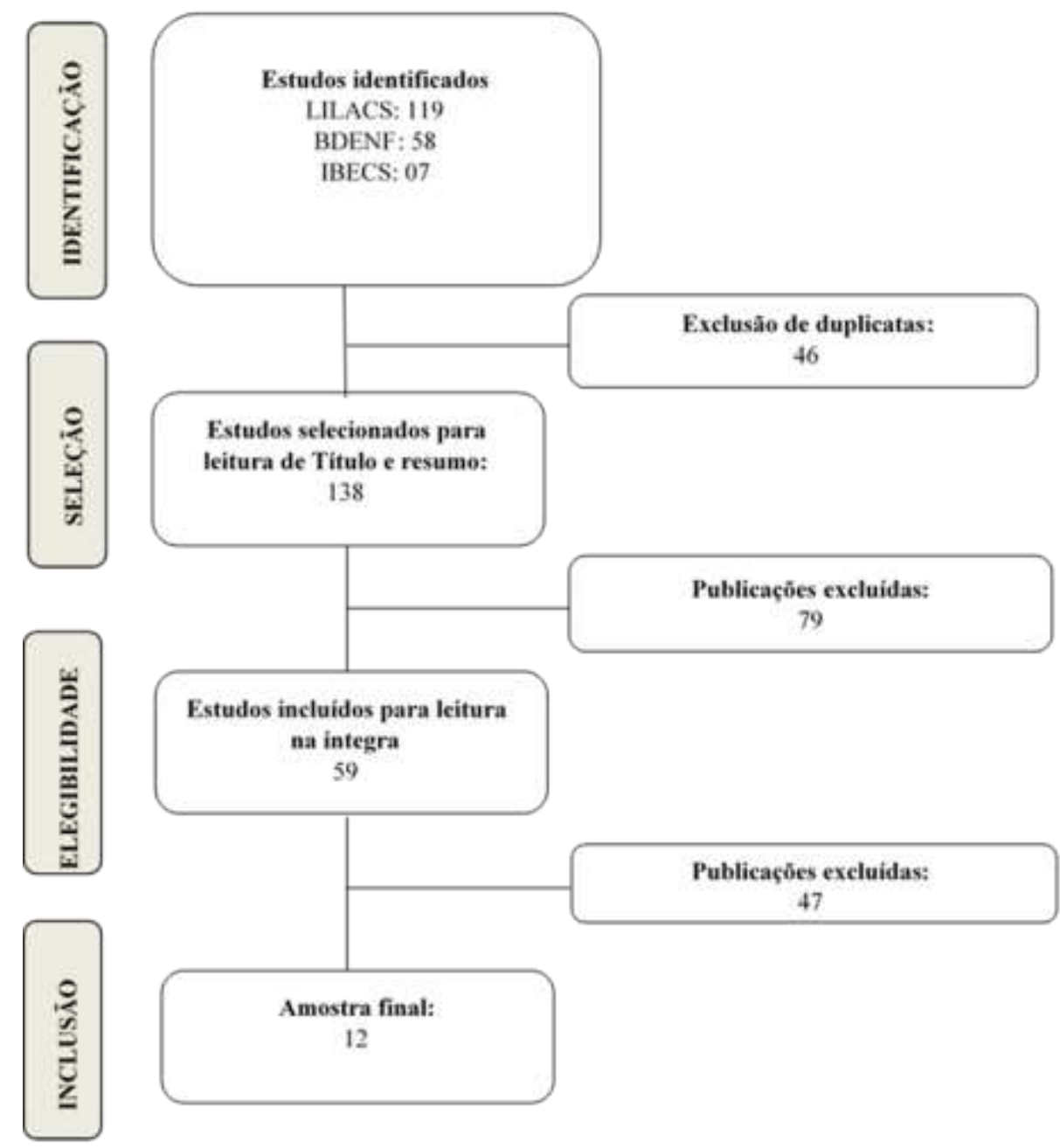

Fonte: Autores.

Este estudo preservou os aspectos éticos de tal forma que todos os autores das publicações analisadas foram referenciados apropriadamente, mediante a Lei de Direitos Autorais nº 9.610 de 19 de Fevereiro de 1998 (Brasil, 1998).

\section{Resultados}

Houve a agregação de doze artigos que atenderam aos critérios para inclusão propostos para esta revisão de literatura. Conforme explicitado, para esta revisão foi construído, pelos autores, um instrumento para coleta de dados. Esses dados são demonstrados compreendendo cada artigo selecionado e suas respectivas informações (Quadro 2).

Observa-se que a frequência de publicação por ano foram: 2015 (n: 04), 2016 (n: 02), 2017 (n: 01), 2018 (n: 03), 2019 (n: 01) e 2020 (n: 01). Em relação aos Qualis dos periódicos da amostra, conforme dados da Coordenação de Aperfeiçoamento de Pessoal de Nível Superior (CAPES), a frequência foi a seguinte: B1 (n: 05), B2 (n: 03), B3 (n: 02) e sem Qualis (n: 02).

Referente à abordagem dos artigos encontrados, verifica-se que a maior parte utilizou o estudo transversal (n: 11) e 
apenas um estudo foi do tipo coorte, ou seja, o nível de evidência mais comum presente nos estudos realizados foi o fraco (VI), embora o estudo de coorte seja nível moderado (III).

Quanto ao local de realização do estudo, observa-se as seguintes distribuições por região do Brasil: Nordeste (n: 05), Sul (n: 03), Sudeste (n: 03), Norte (n: 02) e Centro Oeste (n: 01), com destaque para a publicação de Silva et al. (2015) que fez a análise em todos os Estados da região Nordeste e Norte.

Quadro 2. Caracterização geral dos artigos da amostra, Recife (PE), Brasil, 2020.

\begin{tabular}{|c|c|c|c|c|c|}
\hline $\begin{array}{l}\mathrm{N}^{0} \text { de } \\
\text { ordem }\end{array}$ & $\begin{array}{l}\text { Autores } \\
\text { (ano) }\end{array}$ & $\begin{array}{l}\text { Periódico } \\
\text { (Qualis) }\end{array}$ & $\begin{array}{l}\text { Estado de } \\
\text { realização do } \\
\text { estudo }\end{array}$ & Objetivos & $\begin{array}{l}\text { Tipo de } \\
\text { estudo } \\
\left(\mathrm{NE}^{*}\right)\end{array}$ \\
\hline 01 & $\begin{array}{l}\text { Bastian et al. } \\
\text { (2015) }\end{array}$ & $\begin{array}{c}\text { Nutrire Rev. } \\
\text { Soc. Bras. } \\
\text { Aliment. Nutr } \\
\text { (B3) }\end{array}$ & $\begin{array}{l}\text { Rio Grande do } \\
\text { Sul }\end{array}$ & $\begin{array}{l}\text { Verificar o tempo de AM e os fatores de } \\
\text { risco para o desmame precoce em crianças. }\end{array}$ & $\begin{array}{c}\text { Transversal } \\
\text { (VI) }\end{array}$ \\
\hline 02 & $\begin{array}{l}\text { Schincaglia } \\
\text { et al. (2015) }\end{array}$ & $\begin{array}{l}\text { Epidemiol. serv. } \\
\text { saúde (B2) }\end{array}$ & Goiás & $\begin{array}{l}\text { Analisar os fatores associados à introdução } \\
\text { precoce da alimentação complementar entre } \\
\text { crianças menores de } 6 \text { meses. }\end{array}$ & $\begin{array}{c}\text { Transversal } \\
\text { (VI) }\end{array}$ \\
\hline 03 & $\begin{array}{l}\text { Pellegrinelli } \\
\text { A.L.R. } \\
\text { (2015) }\end{array}$ & $\begin{array}{l}\text { Rev. Nutr. } \\
\text { (Online)** }\end{array}$ & Minas Gerais & $\begin{array}{l}\text { Avaliar o uso de chupeta e mamadeira e sua } \\
\text { influência na prevalência do AME. }\end{array}$ & $\begin{array}{c}\text { Transversal } \\
\text { (VI) }\end{array}$ \\
\hline 04 & $\begin{array}{l}\text { Teter M.S.H. } \\
\text { (2015) }\end{array}$ & $\begin{array}{l}\text { Espaç. saúde } \\
(\text { Online })^{* *}\end{array}$ & Paraná & $\begin{array}{l}\text { Identificar os fatores que levam ao desmame } \\
\text { precoce em Curitiba. }\end{array}$ & $\begin{array}{c}\text { Transversal } \\
\text { (VI) }\end{array}$ \\
\hline 05 & $\begin{array}{l}\text { Moraes } \\
\text { K.A.F. } \\
\text { (2016) }\end{array}$ & $\begin{array}{l}\text { Pesqui. bras. } \\
\text { odontopediatria } \\
\text { clín. integr (B2) }\end{array}$ & $\begin{array}{c}\text { Minas Gerais e } \\
\text { Bahia }\end{array}$ & $\begin{array}{l}\text { Identificar os fatores que influenciaram o } \\
\text { desmame precoce e sua incidência em } \\
\text { crianças. }\end{array}$ & $\begin{array}{c}\text { Transversal } \\
\text { (VI) }\end{array}$ \\
\hline 06 & $\begin{array}{l}\text { Margotti E. } \\
\text { (2016) }\end{array}$ & $\begin{array}{l}\text { Rev. RENE } \\
\text { (B1) }\end{array}$ & $\begin{array}{l}\text { Rio Grande do } \\
\text { Sul }\end{array}$ & $\begin{array}{l}\text { Determinar os fatores de risco para o } \\
\text { desmame precoce. }\end{array}$ & Coorte (III) \\
\hline 07 & $\begin{array}{l}\text { Silva C.S. } \\
\text { (2017) }\end{array}$ & J. pediatr. (B1) & $\begin{array}{l}\text { Estados do } \\
\text { Nordeste e } \\
\text { Norte }\end{array}$ & $\begin{array}{l}\text { Verificar a associação entre a depressão pós- } \\
\text { parto e a ocorrência do AME. }\end{array}$ & $\begin{array}{c}\text { Transversal } \\
\text { (VI) }\end{array}$ \\
\hline 08 & $\begin{array}{l}\text { Margotti E. } \\
\text { (2018) }\end{array}$ & $\begin{array}{l}\text { Rev. enferm. } \\
\text { atenção saúde } \\
\text { (B3) }\end{array}$ & Pará & $\begin{array}{l}\text { Apresentar os índices de AME e verificar os } \\
\text { fatores associados ao desmame aos quatro } \\
\text { meses, em mães adolescentes. }\end{array}$ & $\begin{array}{c}\text { Transversal } \\
\text { (VI) }\end{array}$ \\
\hline 09 & Santos P.V. & Rev. eletrônica & Teresina & Avaliar os fatores associados ao desmame & Transversal \\
\hline
\end{tabular}


Research, Society and Development, v. 10, n. 1, e58010111630, 2021

(CC BY 4.0) | ISSN 2525-3409 | DOI: http://dx.doi.org/10.33448/rsd-v10i1.11630

\begin{tabular}{|c|c|c|c|c|c|}
\hline & $(2018)$ & enferm (B1) & & precoce na Estratégia de Saúde da Família. & $(\mathrm{VI})$ \\
\hline 10 & $\begin{array}{l}\text { Batista } \\
\text { C.L.C. } \\
\text { (2018) }\end{array}$ & J. pediatr. (B1) & Maranhão & $\begin{array}{l}\text { Investigar a associação entre uso de chupeta } \\
\text { e mamadeira e comportamentos } \\
\text { desfavoráveis à amamentação durante as } \\
\text { mamadas. }\end{array}$ & $\begin{array}{c}\text { Transversal } \\
\text { (VI) }\end{array}$ \\
\hline 11 & $\begin{array}{l}\text { Silva V.A.L. } \\
\qquad(2019)\end{array}$ & J. pediatr. (B1) & Pernambuco & $\begin{array}{l}\text { Analisar os fatores associados ao aleitamento } \\
\text { materno exclusivo em um aglomerado } \\
\text { 1urbano subnormal (favela) em Pernambuco }\end{array}$ & $\begin{array}{c}\text { Transversal } \\
\text { (VI) }\end{array}$ \\
\hline 12 & $\begin{array}{l}\text { Carrascoza } \\
\text { K.C. }(2020)\end{array}$ & $\begin{array}{l}\text { Estud. psicol. } \\
\qquad \text { (B2) }\end{array}$ & São Paulo & $\begin{array}{l}\text { Identificar fatores que influenciam a } \\
\text { ocorrência do desmame precoce e do } \\
\text { aleitamento prolongado }\end{array}$ & $\begin{array}{c}\text { Transversal } \\
\text { (VI) }\end{array}$ \\
\hline
\end{tabular}

Nota: *NE - Nível de evidência; ** Sem registro de qualis na CAPES.

Fonte: Dados obtidos no estudo.

Conforme é explicitado no Quadro 3, os principais fatores associados ao desmame precoce encontrado nos estudos brasileiros foram uso da chupeta, escolaridade materna, crença materna na falta de leite, trabalhar fora do lar, uso da mamadeira, tabagismo materno, ser primípara, classe econômica e estado civil. Todos esses fatores estavam associados estatisticamente, conforme descrição dos estudos. 
Quadro 3. Fatores associados ao desmame precoce em estudos brasileiros, Recife (PE), Brasil, 2020.

\begin{tabular}{|c|c|c|}
\hline $\begin{array}{l}\mathrm{N}^{0} \text { de } \\
\text { ordem }\end{array}$ & Fatores associados (associação estatística) & $\mathbf{N}$ total (\% de desmame precoce) \\
\hline 01 & Uso de chupeta nos primeiros dias $\left(\mathrm{OR}^{*}: 2,30 ; \mathrm{p}=0,030\right)$ & $55(1,8 \%)$ \\
\hline 02 & Tabagismo materno $\left(\mathrm{OR}^{*}: 1,02\right)$; primíparas $(\mathrm{OR}: 0,97)$ & $362(42,9 \%)$ \\
\hline 03 & Uso da mamadeira (OR*: 0,43$)$ & $9474(39,4 \%)$ \\
\hline 04 & Escolaridade materna $\left(\mathrm{OR}^{*}: 2,4588\right)$ & $120(17,5 \%)$ \\
\hline 05 & Crença materna na falta de leite $(\mathrm{p}<0,001)$ & $104(30 \%)$ \\
\hline 06 & $\begin{array}{l}\text { Trabalhar fora do lar }(\mathrm{p}=0,013 \text { e } \mathrm{p}=0,007) \text {; escolaridade materna }(\mathrm{p}= \\
\qquad 0,004)\end{array}$ & $300(51 \%)$ \\
\hline 07 & Depressão pós-parto $\left(\mathrm{OR}^{*}=1,67 ; \mathrm{p}=<0,001\right)$ & $(49,2 \%)$ \\
\hline 08 & Trabalhar fora do lar $\left(\mathrm{OR}^{*}: 1,061 ; \mathrm{p}=0,000\right)$ & $92(17,4 \%)$ \\
\hline 09 & Classe econômica $(p=0,000)$ & $241(20,57 \%)$ \\
\hline 10 & Uso de chupeta $(\mathrm{p}=<0,001)$ & 427 \\
\hline 11 & Estado civil (mãe solteira) $(\mathrm{p}=0,0078)$ & $310(32,9 \%)$ \\
\hline 12 & Uso de chupeta nos primeiros dias $\left(\mathrm{OR}^{*}: 2,30 ; \mathrm{p}=0,030\right)$ & $80(83,3 \%)$ \\
\hline
\end{tabular}

Nota: ${ }^{\ddagger}$ OR (Odds Ratio - Razão de chance)

Fonte: Dados obtidos no estudo.

\section{Discussão}

Esta revisão reuniu evidências acerca dos fatores associados ao desmame precoce no período alusivo aos seis primeiros meses de vida da criança, gerando um escopo sólido de evidências científicas a fim de corroborar com a comunidade científica acerca da prevenção dos fatores modificáveis que podem ser maléficos tanto a lactante quanto a lactente.

À luz dos resultados, verificou-se que o fator associado ao desmame precoce mais frequente listado foi o uso de chupeta. Um estudo de revisão sistemática recente, cuja análise foi de 20 estudos internacionais, demonstrou que a chupeta usada em crianças em AME, aumentou o risco em 2 vezes de apresentar desmame precoce (Buccini et al., 2017).

As razões para a introdução de uma chupeta precisam ser determinadas. Ao longo de gerações seu uso se remete a um fator cultural, onde é utilizada para acalmar a criança em crises de choros. Passou a ser contraindicada ao passar dos anos, não só por provocar o confundimento de bicos e prejudicar o estabelecimento da amamentação, mas também pelo fato de postergar a mamada, ao ser empregada para acalmar a criança, que, na realidade, está faminta, favorecendo o desmame (Santos et al., 2019).

Quanto menos a criança suga o seio materno, menor é o estímulo à produção de leite. Também é necessário determinar se os problemas de amamentação associados ao uso de chupetas precedem ou seguem sua introdução. No primeiro 
caso, as mulheres precisam ser orientadas sobre como prevenir, identificar e controlar os problemas da amamentação, como forma de diminuir a necessidade de uso de chupeta. No último caso, no entanto, as mulheres precisam ser desencorajadas a introduzir chupetas para reduzir o risco de problemas de amamentação e aumentar a duração da amamentação (Tavoulari et al., 2016; Sampaio et al., 2020).

A mamadeira também foi um fator associado ao desmame precoce antes do $6^{\circ}$ mês de vida, a exemplo dos resultados do estudo realizado por Pellegrinelli et al. (2015) em um banco de leite humano do Brasil, onde a prevalência de uso da mamadeira foi de 22,9\%, de tal modo que seu uso se associou à menor prevalência de aleitamento materno exclusivo. A esse respeito, estudo realizado no Brasil demonstrou que a alimentação com mamadeira interferiu no desenvolvimento orofacial do bebê, principalmente na posição e na ação muscular dos lábios e da língua e eleva o risco de infecção do trato gastrointestinal; além disso, a posição e a sucção inadequada favorecem a extração irregular e insuficiente do leite (Batista et al., 2018; Santos et al., 2019).

Outros estudos encontrados e apoiados nos resultados, associaram que as características sociodemográficas das mães estão associadas à prática da amamentação como primariedade, estado civil, escolaridade materna e condição econômica (Teter et al., 2015; Margotti \& Mattiello, 2016; Margotti \& Margotti, 2018; Santos et al., 2018; Carrascoza et al., 2020).

Com relação a primariedade, verificou-se que mulheres primíparas têm maior probabilidade de desmamar precocemente a criança em AME. Em uma pesquisa foi constatado que mulheres primíparas apresentaram maiores riscos de desmamar precocemente do que as multíparas. Os autores do estudo justificam tal resultado ao fato das mulheres primíparas terem tido menor oportunidade de acesso às informações veiculadas na mídia e às ações de promoção do aleitamento materno do que as mães com mais filhos (Schincaglia et al., 2015).

No que diz respeito ao estado civil verificou-se menor prevalência do aleitamento materno exclusivo ente as mulheres solteiras. Um estudo realizado por Silva et al. (2019) verificou que o apoio do companheiro é importante para superar as dificuldades presentes no ato de amamentar, isto porque as mulheres que têm família estável, que moram com companheiros, amamentam significativamente mais tempo do que as solteiras.

A escolaridade materna também foi um preditor para o desmame precoce. O baixo nível de escolaridade da mãe pode contribuir como fator negativo para a execução da amamentação. Devido à baixa escolaridade das mães, provavelmente, elas acabam por não ter acesso às informações e aos serviços de saúde que possibilitem entender melhor sobre os efeitos benéficos que o aleitamento materno proporciona para a saúde da criança e da mãe (Cunha et al., 2016).

Mulheres mais jovens e principalmente aquelas que vivem em áreas mais carentes têm menos qualificações e menos probabilidade de iniciar a amamentação e mais probabilidade de manter apenas um curto período de amamentação exclusiva (Teter et al., 2015).

À luz dos resultados evidenciou-se ainda que o tabagismo influencia o processo de desmame precoce. As mulheres que fumam podem desmamar prematuramente por não terem certeza se ainda é seguro amamentar. Essas mulheres podem relutar em procurar aconselhamento de profissionais de saúde, ou mesmo ajuda para problemas de amamentação (Tavoulari et al., 2016). Um estudo de revisão sistemática conduzido por Santana et al. (2018) analisou que o uso do tabagismo pode influenciar negativamente na amamentação evidenciando a necessidade de maiores informações à população e aos profissionais de saúde sobre seus efeitos negativos.

Mediante os resultados expostos por Schincaglia et al., (2015) o fato da mãe ser primípara também influenciou no processo de desmame precoce. De acordo com Oliveira et al. (2015), as práticas culturais de introdução precoce de chás, água e alimentos junto com o leite materno, provavelmente têm um impacto maior nas práticas com o primeiro filho quando as mães não têm um bom suporte para a amamentação exclusiva. Assim, mães com experiência anterior no processo de amamentação e possivelmente mais velhas, costumam ser mais maduras no que diz respeito aos cuidados e alimentação de um filho quando 
comparadas às primíparas.

Muitos destes fatores associados ao desmame precoce são passíveis de intervenção, a exemplo das crenças maternas na "falta de leite". A cessação da amamentação está relacionada às percepções das mães, potencialmente, ela poderia ser abordada com mais informações que enfatizem as dificuldades que podem ocorrer no início da amamentação. A percepção do baixo suprimento de leite pode ser devido à falta de confiança na amamentação e no suprimento de leite, e deve ser tratada com apoio e educação, em vez de enfatizar os problemas (Tadele et al., 2016).

Percebe-se então que intervenções relacionadas à educação são essenciais para o estímulo ao aleitamento materno exclusivo, devendo ser realizadas tanto no pré-natal, como na sala de parto, no alojamento conjunto e no puerpério. Além disso a rede de apoio familiar também precisa receber estas orientações de modo que uma mãe que não amamenta facilmente perde a confiança em si mesma e fica suscetível à pressão de parentes e amigos, além de transmitir esse sofrimento para outras nutrizes.

Neste sentido o enfermeiro é o profissional mais capacitado para educar e orientar tanto a mãe como sua rede familiar, podendo atuar junto à população, não somente prestando assistência, mas também na promoção e educação continuada, de forma efetiva (Araújo et al., 2020). Estudo qualitativo conduzido por Oliveira et al. (2017) constatou que segundo a visão das mulheres entrevistadas o enfermeiro configurou-se como um agente facilitador no incentivo e manejo do aleitamento materno.

Tudo isto deve ser pautado no princípio da promoção da saúde, que deve propiciar, sobretudo, o fortalecimento das ações comunitárias e o desenvolvimento de habilidades pessoais da mãe, a aspectos inerentes ao aleitamento materno.

\section{Conclusão}

A literatura analisada evidenciou que os fatores associados ao desmame precoce mais frequentes no Brasil estão associados a hábitos e crenças como o uso da chupeta e da mamadeira, e a aspectos socioeconômicos como escolaridade materna e classe social, estado civil, condições de trabalho, tabagismo materno e mulheres primíparas.

Desta forma, a identificação oportuna e o conhecimento íntimo desses fatores podem ajudar a reconhecer as mulheres em risco de desmame precoce e a construir programas capazes de aumentar a duração, especialmente no âmbito da Atenção Primária à Saúde, onde este trabalho deve se iniciar precocemente no pré-natal.

O profissional enfermeiro tem fundamental importância neste processo, tanto no âmbito do pré-natal como no pósparto e nas consultas de puericultura, atuando como orientador e incentivador para uma boa prática do aleitamento materno, tornando-a um ato de prazer e dedicação, no qual a assistência de enfermagem terá um elo com o conhecimento científico, de modo que poderá garantir os benefícios do aleitamento materno.

Como perspectiva futura, espera-se ampliar o escopo desta temática com estudos mais aprofundados, que permitam realizar estatisticamente os dados fornecidos pelos estudos primários, afim de gerar uma base de evidências sólidas a respeito dos fatores associados ao desmame precoce.

\section{Referências}

Araújo, G. B., Fernandes, A. B., Oliveira, A. C. A., Gomes, E. G. R., Pereira, T. L., \& Oliveira, L. S. et al. (2020). Contribuições do enfermeiro para a promoção do aleitamento materno. Braz. J. Hea. Rev, 3(3), 4841-4863.

Bastian, D. P., \& Terrazzan, A. C. (2015). Tempo de aleitamento materno e os fatores de risco para o desmame precoce. Nutrire Rev. Soc. Bras. Aliment. Nutr; 40(3), 278-286.

Batista, C. L. C., Ribeiro, V. S., Nascimento, M. D. S. B., \& Rodrigues, V. P. (2018). Association between pacifier use and bottle-feeding and unfavorable behaviors during breastfeeding. J. pediatr. (Rio J.); 94(6), 596-601.

Buccini, G. S., Pérez-Escamilla, R., Paulino, L. M., Araújo, C. L., \& Venancio S. I. (2017). Pacifier use and interruption of exclusive breastfeeding: Systematic review and meta-analysis. Matern Child Nutr. 13(3), e12384. 
Carrascoza, K. C., Júnior, A. L. C., \& Moraes, A. B. A. (2020). Fatores que influenciam o desmame precoce e a extensão do aleitamento materno. Estud. psicol. (Campinas); 22(4), 433-440.

Cunha, M. C. D., Macedo, P. T. T. R., Rocha, F. C. G., Souza, J. M. L., Carvalho, M. L., \& Penha, K. J. S. (2016). Desmame precoce entre mulheres na unidade básica de saúde de São Luís -MA. Revista Interdisciplinar, 9(4), 67-73.

D’Auria, E., Bergamini, M., Staiano, A., Banderali, G., Pendezza, E., Penagini, F., et al. (2018). Baby-led weaning: what a systematic review of the literature adds on. Ital J Pediatr. 44, 2-11.

Feitosa, M. E. B., Silva, S. E. O., \& Silva, L. L. (2020). Breastfeeding: causes and consequences of early weaning. Research, Society and Development; 9(7), e856975071.

Lopes, L. M. (2016). Desmame precoce. Trabalho de Conclusão de Curso; Universidade do Estado do Rio de Janeiro. https://ares.unasus.gov.br/acervo/html/ARES/7965/1/L\%C3\%ADvia\%20Maia\%20Lopes.pdf

Margotti, E., \& Margotti, W. (2018). Fatores de risco para o desmame aos quatro meses em bebês de mães adolescentes. Rev. enferm. atenção saúde; 7(3), $116-128$.

Margotti, E., \& Mattiello, R. (2016). Fatores de risco para o desmame precoce. Rev. RENE; 17(4), 537-544.

Ministério da Saúde (MS). (1998). Lei no 9.610, de 19 de fevereiro de 1998, que altera, atualiza e consolida a legislação sobre direitos autorais e dá outras providências. Diário Oficial da União. Brasília: Ministério da Saúde. http://www.planalto.gov.br/ccivil_03/leis/19610.htm

Ministério da Saúde (MS). (2015). Technical Document on the Regulation of the Publicity and Labeling of Products that Interfere on Breastfeeding. https://www.paho.org/hq/dmdocuments/2015/breastfeeding-protection-technical-doc-

decree.pdf\#: :text=In\%20Brazil\%2C\%20exclusive\%20breastfeeding\%20rates,from\%20attending\%20the\%20WHO\%20recommendations

Oliveira, A. K. P., Melo, R. A., Maciel, L. P., Tavares, A. K., Amando, A. R., \& Sena, C. R. S. (2017). Práticas e crenças populares associadas ao desmame precoce. Av Enferm. 35(3), 303-312.

Oliveira, C. S., Locca, F. A., Carrijo, M. L. R., \& Garcia, R. A. T. M. (2015). Amamentação e as intercorrências que contribuem para o desmame precoce. Rev Gaúcha Enferm. 36, 16-23.

Pellegrinelli, A. L. R., Pereira, S. C. L., Ribeiro, I. P., \& Santos, L. C. (2015). Influência do uso de chupeta e mamadeira no aleitamento materno exclusivo entre mães atendidas em um Banco de Leite Humano. Rev. Nutr. (Online); 28(6), 631-639.

Pereira, A. S., Shitsuka, D. M., Parreira, F. J., Shitsuka, R. Metodologia da pesquisa científica. (1 ${ }^{\text {a }}$ ed.) -: UFSM, NTE, 2018. https://repositorio.ufsm.br/bitstream/handle/1/15824/Lic_Computacao_Metodologia-Pesquisa-Cientifica.pdf?sequence=1

Pereira, M. G. (2011). Artigos científicos: como redigir, publicar e avaliar. Rio de Janeiro: Guanabara Koogan.

Sampaio, R. C. T., Brito, M. B. G., Siebra, L. G. B., Gonçalves, G. K. M., Feitosa, D. M. A., Cabral, K. S. S. A., et al. (2020). Associação entre o uso de chupetas e interrupção da amamentação: Uma revisão de literatura. Braz. J. Hea. Rev. 3(4), 7353-7372.

Santana, G. S., Giugliani, E. R. J., Vieira, T. O., \& Vieira, G. O. (2018). Fatores associados à manutenção da amamentação por 12 meses ou mais: revisão sistemática. J. Pediatr. (Rio J.); 94(2), 104-122.

Santos, E. M., Silva, L. S., Rodrigues, B. F. S., Amorim, T. M. A. X., Silva, C. S., Borba, J. M. C., et al. (2019). Avaliação do aleitamento materno em crianças até dois anos assistidas na atenção básica do Recife, Pernambuco, Brasil. Ciênc. saúde colet. 24(3), 1211-1222.

Santos, P. V., Martins, M. C. C., Tapety, F. I., Paiva, A. A., Fonseca, F. M. N. S., \& Brito. A. K. S. (2018). Desmame precoce em crianças atendidas na EstrateÌgia Sauİde da Famil̀lia. Rev. Eletr. Enf.; 20(5), 1-12.

Schincaglia, R. M., Oliveira, A. C., Sousa, L. M., \& Martins, K. A. (2015). Práticas alimentares e fatores associados à introdução precoce da alimentação complementar entre crianças menores de seis meses na região noroeste de Goiânia. Epidemiol. serv. saúde; 24(3), $465-474$.

Silva, C. S., Lima, M. C., Sequeira-de-Andrade, L. A. S., Oliveira, J. S., Monteiro, J. S., Lima, N. M. S., et al. (2017). Associação entre a depressão pós-parto e a prática do aleitamento materno exclusivo nos três primeiros meses de vida. J. pediatr. (Rio J.); 93(4), $356-364$.

Silva, V. A. A. L., Caminha, M. F. C., Silva, S. L., Serva, V. M. S. B. D., Azevedo, P. T. A. C. C. \& Filho, M. B. (2019). Aleitamento materno: indicadores e fatores associados à amamentação exclusiva num aglomerado urbano subnormal assistido pela Estratégia de Saúde da Família. J. pediatr.; 95(3), 298-305.

Souza, M. A. O., Souza, N. R., Melo, J. T. S., Xavier, M. A. C. A., Almeida, G. L., \& Santos, I. C. R. V. (2018). Odor evaluation scales for odor in neoplastic wounds: an integrative review. Rev Bras Enferm. 71(5), 2552-2560.

Souza, M. T., Silva, M. D., \& Carvalho, R. (2010). Integrative review: what is it? How to do it? Einstein (São Paulo). 8(1), $102-106$.

Stillwell, S., Fineout-Overholt, E., Melnyk, B. M., \& Wiliamson, K. M. (2010). Evidence- based practice: step by step. Am J Nurs; $110(5)$, $41-47$.

Tadele, N., Habta, F., Akmel, D., \& Deges, E. (2016). Knowledge, attitude and practice towards exclusive breastfeeding among lactating mothers in Mizan Aman town, Southwestern Ethiopia: descriptive cross-sectional study. Int Breastfeed J.; 11(3).

Tavoulari, E-F., Benetou, V., Vlastarakos, P. V., Psaltopoulou, T., Chrousos, G., Kreatsas, G., et al. (2016). Factors affecting breastfeeding duration in Greece: What is important? World J Clin Pediatr. 5(3), 349-357.

Teter, M. S. H., Oselame, G. B., \& Neves, E. B. (2015). Amamentação e desmame precoce em lactantes de Curitiba. Espaç. saúde (Online); 16(4), 54-63. 
Research, Society and Development, v. 10, n. 1, e58010111630, 2021

(CC BY 4.0) | ISSN 2525-3409 | DOI: http://dx.doi.org/10.33448/rsd-v10i1.11630

Victora, C. G., Aluísio, J. D., Barros, A. J. D., França, G. V. A., Horton, S., \& Krasevec, J. (2016). Breastfeeding in the 21st century: Epidemiology, mechanisms, and lifelong effect. Lancet, 387, 475-490.

World Health Organization - WHO. (2018). Global breastfeeding scorecard, 2018: enabling women to breastfeed through better policies and programmes. https://www.who.int/nutrition/publications/infantfeeding/global-bf-scorecard-2018.pdf?ua=1

World Health Organization - WHO. (2020). Breastfeeding. https://www.who.int/health-topics/breastfeeding\#tab=tab_1 